\title{
Interleukin-23R rs7517847 T/G Polymorphism Contributes to the Risk of Crohn's Disease in Caucasians: A Meta-Analysis
}

\author{
Li Zhang, ${ }^{1}$ Yunjie Lu, ${ }^{2}$ Yuzheng Ge, ${ }^{3}$ Yun Shi, ${ }^{1}$ Xing Wu, ${ }^{1}$ Qinghua Xu, ${ }^{1}$ Xiaoping Li, \\ Ling $\mathrm{Lu}^{2}{ }^{2}$ Feng Zhang, ${ }^{2}$ and Guozhong Yao ${ }^{1}$ \\ ${ }^{1}$ Liyang People's Hospital, Liyang 213300, China \\ ${ }^{2}$ Translational Medicine Research Center of Jiangning Hospital and Liver Transplantation Center of First Affiliated Hospital, \\ Nanjing Medical University, Nanjing 210029, China \\ ${ }^{3}$ Department of Urology, Nanjing First Hospital, Nanjing Medical University, Nanjing 210029, China
}

Correspondence should be addressed to Feng Zhang; zhangfeng1958@hotmail.com and Guozhong Yao; 13301498818@163.com

Received 27 June 2014; Revised 5 January 2015; Accepted 12 January 2015

Academic Editor: Guixiu Shi

Copyright (C) $2015 \mathrm{Li}$ Zhang et al. This is an open access article distributed under the Creative Commons Attribution License, which permits unrestricted use, distribution, and reproduction in any medium, provided the original work is properly cited.

\begin{abstract}
The association between Interleukin-23R gene polymorphism and Crohn's disease (CD) in Caucasians is still controversial. Thus, a meta-analysis was performed to evaluate the correlation between this gene variant and CD risk. We retrieved the available data from EMBASE and PUBMED until May 1, 2014, and evaluated the effect of rs7517847 in Caucasians. The significant associations were confirmed between rs7517847 and CD risk in dominant models (TT/TG versus GG: OR = 1.652, 95\% CI 1.277, 2.137), allelic model ( $\mathrm{T}$ allele versus $\mathrm{G}$ allele: $\mathrm{OR}=1.327,95 \% \mathrm{CI} 1.198,1.469$ ), homozygote comparison (TT versus GG: OR = 1.890, $95 \% \mathrm{CI}$ 1.465, 2.437), heterozygote comparison (TG versus GG: $\mathrm{OR}=1.509,95 \% \mathrm{CI} 1.161,1.960$ ), and recessive model (TT versus TG/GG: $\mathrm{OR}=1.409,95 \% \mathrm{CI} 1.279,1.552)$. In conclusion, this meta-analysis demonstrates that rs7517847 is associated with the risk of CD in Caucasians. These findings show that IL-23R genes confer susceptibility to CD in the Caucasians.
\end{abstract}

\section{Introduction}

Crohn's disease $(\mathrm{CD})$ is a form of inflammatory bowel disease (IBD) that primarily affects the Caucasian population $[1,2]$. It is a heritable disease which is influenced by many genetic risk factors [3]. Therefore, identification of gene risk factors of $\mathrm{CD}$ is beneficial for the clinical treatment of patients.

Interleukin 23 (IL-23) plays an important role in the inflammatory response against infection as a regulator of immune cells [4]. IL-23R which interacts with IL-23 is a protein consisting of an IL-12 $\beta 1$ and an IL-23R chain [5]. Recently, the mechanisms of IL-23R variants have been investigated in different autoimmune diseases [6-9]. Studies also have shown that rs7517847, the single nucleotide polymorphisms (SNPs) of the IL23R gene, are associated with CD occurring rate $[10,11]$. However, the association between IL23R polymorphisms and CD susceptibility are inconclusive and controversial due to small sample size in each of the published studies.

To better understand the association of IL-23R polymorphisms and CD susceptibility in Caucasians, we conducted a meta-analysis of all eligible studies and hope to yield more accurate and robust estimates.

\section{Materials and Methods}

2.1. Search Strategy. We searched for relevant studies in the following databases: EMBASE and PUBMED. Available studies for IL-23R polymorphism and CD were collected by different combinations of various key words: Interleukin23 receptor, IL-23R; polymorphism, variant, or mutation; Crohn's disease, CD. Languages restriction was not imposed in this research and only published studies with full text were included in this meta-analysis.

2.2. Inclusion and Exclusion Criteria of Trials. In the metaanalysis, the following inclusive selection criteria were set: (a) study design evaluating the association between IL-23R polymorphism and CD risk; (b) case control design; (c) Caucasians design. The following exclusive selection criteria were set: (a) no control cases; (b) duplication of the previous 


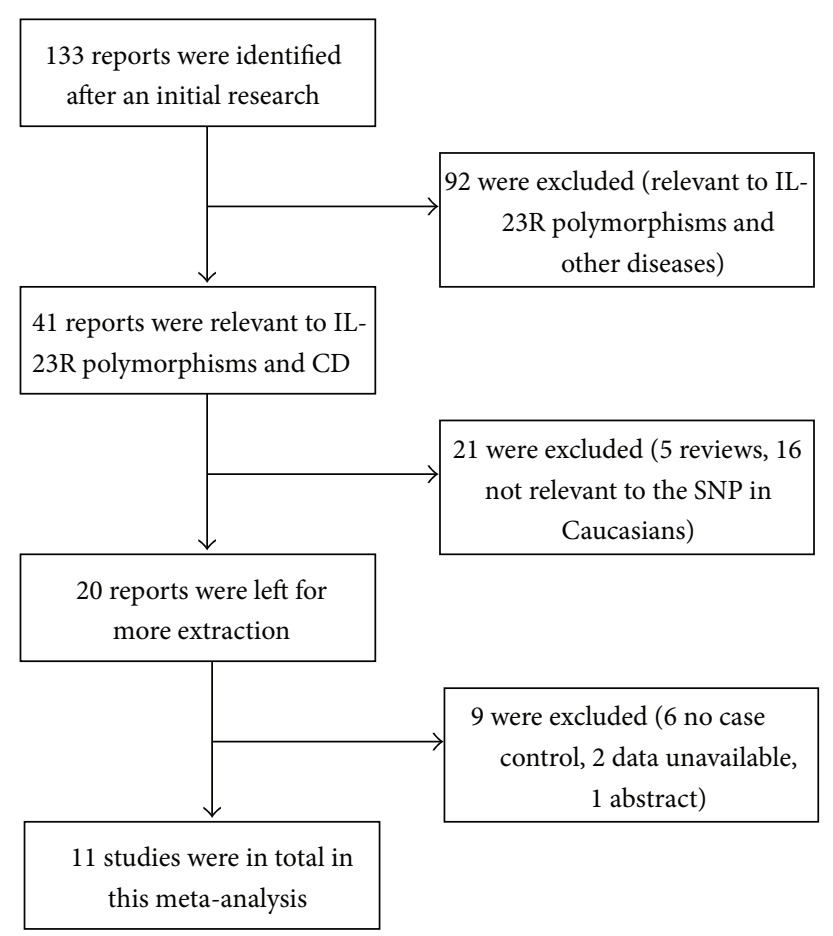

FIgURE 1: Flow diagram of the study selection process and specific reasons for exclusion.

publication; (c) no available genotype frequency; for studies with overlapped or repeated data (d) no Caucasians.

2.3. Data Extraction. Eligible studies were extracted by 2 reviewers (Li Zhang and Yunjie Lu) independently according to the predesigned data collection form. The following information was extracted: first author's name, publication year, country, ethnicity, immune suppressive protocol, number of cases and controls, and genotype distribution in both groups. Disagreement was resolved by discussion with a third reviewer (Guozhong Yao).

2.4. Statistical Analysis. For each trial, odds ratio (OR) with the $95 \%$ confidence interval ( $95 \% \mathrm{CI}$ ) of the survival rate was derived and calculated. Increased or decreased risk of CD was indicated by $95 \%$ CI without 1 for OR. The pooled ORs were estimated for allelic model ( $T$ allele versus $G$ allele), homozygote comparison (TT versus GG) and heterozygote comparison (TG versus GG), dominant models (TT/TG versus GG), and recessive model (TT versus TG+GG). $Z$ test was performed to assess the significance of the pooled OR. Between-study heterogeneity was assessed by the Cochran's $Q$ statistic and $I^{2}$ tests [12]. The random effects model was conducted if the $Q$ test exhibited a $P<0.05$ or the $I^{2}$ test showed $>50 \%$. Otherwise, the fixed effects model would be conducted. For publication bias, the Begg's funnel plot and Egger's linear regression test were conducted, and $P<0.05$ was considered significant.

A fixed-effect model (based on Mantel-Haenszel method) was utilized to pool the data from different studies if the between-study heterogeneity was absent, or a random-effect model (based on DerSimonian and Laird method) was applied.

The statistical analysis was performed by STATA 10.0 (Stata Corp LP, College Station, TX, USA). All $P$ values are two-side.

\section{Results}

3.1. Selection of the Included Studies and Characteristics. The flow diagram of studies selection and exclusion reasons were represented in Figure 1. A total of 133 studies were identified by our first research; a number of 41 were preliminarily yielded out after excluding inappropriate studies and screening abstract-screening, full-text assessment. In these 41 studies, 30 were excluded, 11 articles containing rs7517847 in Caucasians were recruited for detailed analysis (Table 1), and these data built Table 1 [13-23]. Each of them was independent. Thus, a total of $3279 \mathrm{CD}$ cases and 4136 healthy controls were included in our meta-analysis. All of them were Caucasian and the diagnosis of $\mathrm{CD}$ was based on clinical manifestations and laboratory examinations and further biopsy.

3.2. Evaluation of the Association. The OR from all models indicated a significant association between rs7517847 and CD. After pooling all the eligible studies in Table 2, we found that the risk of CD was significantly associated with rs7517847 in dominant models (TT/TG versus GG: $\mathrm{OR}=1.652,95 \% \mathrm{CI}$ $1.277,2.137$ ), allelic model ( $\mathrm{T}$ allele versus $\mathrm{G}$ allele: $\mathrm{OR}=$ $1.327,95 \%$ CI 1.198, 1.469), homozygote comparison (TT versus GG: $\mathrm{OR}=1.890,95 \%$ CI 1.465, 2.437, Figure 2), 
TABLE 1: Characteristics of eligible studies included in the meta-analysis.

\begin{tabular}{|c|c|c|c|c|c|c|c|c|c|c|c|}
\hline \multirow{2}{*}{ Author } & \multirow{2}{*}{ Year } & \multirow{2}{*}{ Country } & \multirow{2}{*}{ Ethnicity } & \multirow{2}{*}{ Genotyping methods } & \multicolumn{3}{|c|}{ Case } & \multicolumn{3}{|c|}{ Control } & \multirow{2}{*}{ HWE } \\
\hline & & & & & $\mathrm{TT}$ & TG & GG & $\mathrm{TT}$ & TG & GG & \\
\hline Safrany et al. [13] & 2013 & Hungary & Caucasian & PCR-RFLP & 72 & 110 & 17 & 74 & 138 & 41 & 0.081 \\
\hline Szabo et al. [14] & 2013 & Hungary & Caucasian & PCR-RFLP & 150 & 182 & 64 & 57 & 99 & 26 & 0.104 \\
\hline Ferguson et al. [15] & 2010 & New Zealand & Caucasian & MassARRAY & 108 & 172 & 29 & 113 & 183 & 72 & 0.892 \\
\hline Lauriola et al. [16] & 2011 & Italy & Caucasian & PCR & 9 & 4 & 6 & 13 & 6 & 1 & 0.78 \\
\hline Lacher et al. [17] & 2010 & Germany & Caucasian & RT-PCR & 81 & 101 & 39 & 78 & 125 & 50 & 0.995 \\
\hline Latiano et al. [18] & 2008 & Italy & Caucasian & PCR-RFLP & 366 & 305 & 52 & 280 & 328 & 108 & 0.459 \\
\hline Márquez et al. [19] & 2008 & Spain & Caucasian & RT-PCR & 145 & 161 & 36 & 192 & 260 & 94 & 0.71 \\
\hline Baptista et al. [20] & 2008 & Brazil & Caucasian & RT-PCR & 59 & 95 & 28 & 79 & 120 & 43 & 0.825 \\
\hline Newman et al. [21] & 2009 & England & Caucasian & MassARRAY & 195 & 204 & 40 & 300 & 436 & 164 & 0.799 \\
\hline Okazaki et al. [22] & 2008 & Canada & Caucasian & RT-PCR & 84 & 91 & 36 & 91 & 157 & 66 & 0.91 \\
\hline Oliver et al. [23] & 2007 & Spain & Caucasian & Taqman PCR & 101 & 119 & 18 & 121 & 153 & 68 & 0.124 \\
\hline
\end{tabular}

TABLE 2: Stratified analysis of rs7517847 polymorphism and CD risk in eligible studies.

\begin{tabular}{|c|c|c|c|c|c|c|}
\hline $\mathrm{r}$ & Year & $\mathrm{T}$ versus & $\begin{array}{l}\text { TT versus GG } \\
\text { OR }(95 \% \text { CI })\end{array}$ & $\begin{array}{l}\text { TG versus GG } \\
\text { OR }(95 \% \mathrm{CI})\end{array}$ & $\begin{array}{c}\mathrm{TT}+\mathrm{TG} \text { versus GG } \\
(95 \% \mathrm{CI})\end{array}$ & $\begin{array}{c}\text { TT versus TG + GG } \\
(95 \% \mathrm{CI})\end{array}$ \\
\hline Total & & & $1.890(1.465,2.437)$ & $1.509(1.161,1.960)$ & $1.652(1.277,2.137)$ & $1.409(1.279,1.552)$ \\
\hline Safrany et al. [13] & 2013 & $357(1.036,1.777)$ & $347(1.223,4.503)$ & $1.922(1.036,3.568)$ & $2.070(1.137,3.769)$ & $1.371(0.923,2.038)$ \\
\hline zabo et al. [14] & 2013 & $102(0.856,1.419)$ & $0.618,1.850)$ & $0.747(0.445,1.253)$ & $0.865(0.528,1.417)$ & $1.337(0.921,1.942)$ \\
\hline & & & 3) & 2.334 & & \\
\hline & & & & & & \\
\hline & & & r & 8) & & 02) \\
\hline & & & 然 & $1.031(1.070,2.804)$ & & \\
\hline & & & $1.972(1.269,3.063)$ & $1.617(1.050,2.489)$ & $1.768(1.172,2.665)$ & $1.357(1.029,1.791)$ \\
\hline & 2008 & & $1.147(0.640,2.055)$ & $1.216(0.704,2.100)$ & $1.188(0.706,2.000)$ & $0.990(0.656,1.492)$ \\
\hline Newman et al. [21] & 2009 & $1.542(1.302,1.827)$ & $2.665(1.805,3.935)$ & $1.918(1.307,2.815)$ & $2.223(1.541,3.207)$ & $1.598(1.265,2.019)$ \\
\hline Okazaki et al. [22] & 2008 & & $1.692(1.024,2.798)$ & $1.918(1.307,2.815)$ & $1.294(0.825,2.029)$ & $1.621(1.122,2.342)$ \\
\hline Oliver et al. [23] & 2007 & $1.515(1.187,1.935)$ & $3.153(1.761,5.648)$ & $2.938(1.658,5.207)$ & $3.033(1.752,5.252)$ & $1.347(0.959,1.891)$ \\
\hline
\end{tabular}

heterozygote comparison (TG versus GG: OR $=1.509,95 \%$ CI 1.161, 1.960), and recessive model (TT versus TG/GG: $\mathrm{OR}=1.409,95 \%$ CI 1.279, 1.552). These data demonstrate that rs7517847 increases the risk of CD among Caucasians with hospital-based studies.

3.3. Publication Bias. Begg's funnel plot and Egger's test were both performed to assess the publication bias of this meta-analysis. The shape of the funnel plots for homozygote comparison models seemed symmetrical (Figure 3 ). Then, the Egger's test was used to provide statistical evidence of funnel plot symmetry. The results still did not suggest any evidence of publication bias. Thus, publication bias was not evident in present meta-analyses.

\section{Discussion}

$\mathrm{CD}$ is associated with JAK2 signaling pathway which is activated by IL-23 and IL-23R receptor [24]. Previous studies suggested that the interruption of IL-23R SNPs might lead to the dysregulation of intestinal inflammation [25]. IL-23R gene variants also play an essential role in the development of many autoimmune diseases such as ankylosing spondylitis
(AS), inflammatory bowel disease (IBD), and systemic lupus erythematosus (SLE) [7, 26, 27]. Therefore, researchers are focusing on observing the relationship between IL-23R gene polymorphisms and the risk of CD. However, the results are conflicting and controversial due to the different races and insufficient sample size. After pooling data for 11 studies in this meta-analysis, our results firstly demonstrate that $\mathrm{T}$ allele of rs7517847 was highly susceptible to CD in Caucasians.

One previous study showed that rs7517847 is a protective factor in rheumatoid arthritis (RA) in European population. Interestingly, RA is a systemic autoinflammatory disease which is associated with PTPN22/C1858T, while the organspecific autoimmune disease CD is not $[28,29]$. Thus, the mechanism of this genetic variant may not play a common role in different autoimmune diseases. More researches are required to observe the exact mechanisms of IL-23R gene polymorphism.

We should also mention the limitations of this metaanalysis. Primarily, all the studies were limited to the Caucasian. The allelic frequencies may be different in other ethnic groups. Secondly, publication bias might occur even if there is no significance in statistical test due to extracting published studies. Ultimately, owing to methodological limitations, 


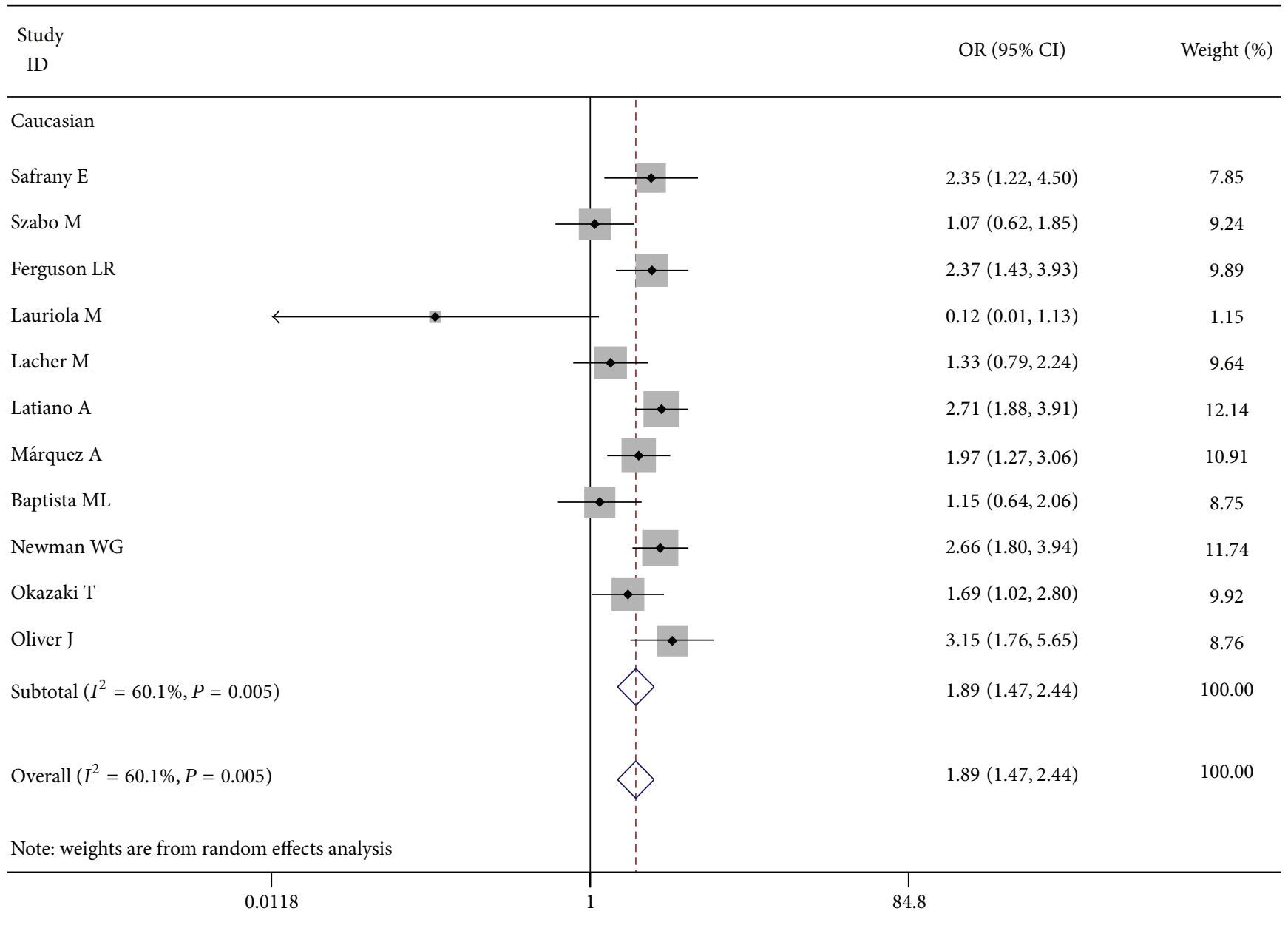

FIGURE 2: Forest plot for risk of CD associated with rs7517847 in Caucasian (TT versus GG. For each study, the estimate of OR and its $95 \%$ CI is plotted with a box and a horizontal line. Filled diamond pooled OR and its 95\% CI).

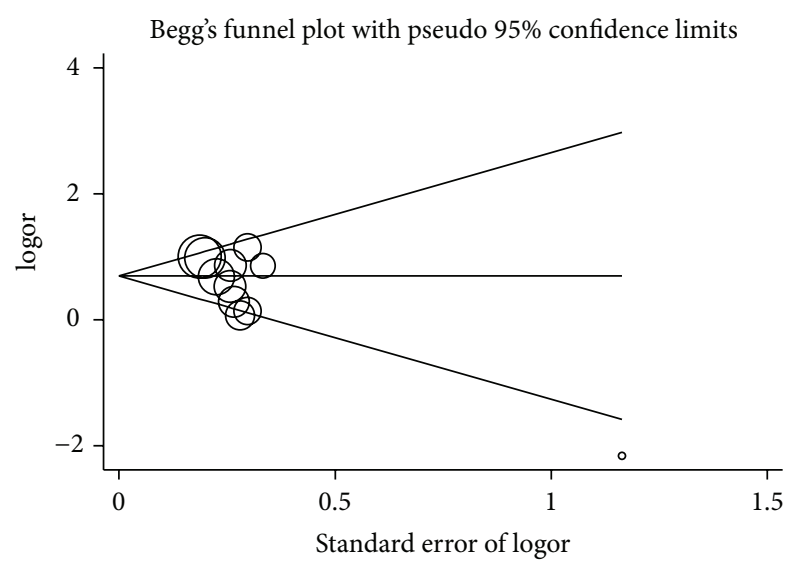

FIGURE 3: Begg's funnel plot for publication bias test (TT versus GG for rs7517847).

this meta-analysis is retrospective. Two independent authors performed the process of study selection and data extraction and a third author resolved the discrepancy to minimize the bias.
In conclusion, our meta-analysis suggests that IL-23R rs7517847 confers susceptibility to CD in the Caucasians. Furthermore, more studies with larger scale are required to confirm these associations.

\section{Conflict of Interests}

All the authors declare that they do not have any commercial or associative interest that represents a conflict of interests in connection with the work submitted.

\section{Authors' Contribution}

Li Zhang and Yunjie Lu contributed equally to this work and both are co-first authors.

\section{Acknowledgment}

This study was supported by the Medical Foundation of Liyang People Hospital. 


\section{References}

[1] H. M. Malaty, J. K. Hou, and S. Thirumurthi, "Epidemiology of inflammatory bowel disease among an indigent multi-ethnic population in the United States," Clinical and Experimental Gastroenterology, vol. 3, no. 1, pp. 165-170, 2010.

[2] E. V. Loftus Jr., "Clinical epidemiology of inflammatory bowel disease: incidence, prevalence, and environmental influences," Gastroenterology, vol. 126, no. 6, pp. 1504-1517, 2004.

[3] J. van Limbergen, D. C. Wilson, and J. Satsangi, “The genetics of Crohn's disease," Annual Review of Genomics and Human Genetics, vol. 10, pp. 89-116, 2009.

[4] C. Parham, M. Chirica, J. Timans et al., "A receptor for the heterodimeric cytokine IL-23 is composed of IL-12R $\beta 1$ and a novel cytokine receptor subunit, IL-23R," Journal of Immunology, vol. 168, no. 11, pp. 5699-5708, 2002.

[5] S. Aggarwal, N. Ghilardi, M.-H. Xie, F. J. De Sauvage, and A. L. Gurney, "Interleukin-23 promotes a distinct CD4 T cell activation state characterized by the production of interleukin17,' The Journal of Biological Chemistry, vol. 278, no. 3, pp. 19101914, 2003.

[6] X. Wang, J. Huang, Z. Lin et al., "Single-nucleotide polymorphisms and expression of IL23R in Chinese ankylosing spondylitis patients," Rheumatology International, vol. 30, no. 7, pp. 955-959, 2010.

[7] R. H. Duerr, K. D. Taylor, S. R. Brant et al., "A genome-wide association study identifies IL23R as an inflammatory bowel disease gene," Science, vol. 314, no. 5804, pp. 1461-1463, 2006.

[8] B. Faragó, L. Magyari, E. Sáfrány et al., "Functional variants of interleukin-23 receptor gene confer risk for rheumatoid arthritis but not for systemic sclerosis," Annals of the Rheumatic Diseases, vol. 67, no. 2, pp. 248-250, 2008.

[9] M. Cargill, S. J. Schrodi, M. Chang et al., "A large-scale genetic association study confirms IL12B and leads to the identification of IL23R as psoriasis-risk genes," American Journal of Human Genetics, vol. 80, no. 2, pp. 273-290, 2007.

[10] H. Dong, Q. Li, Y. Zhang, W. Tan, and Z. Jiang, "IL23R gene confers susceptibility to ankylosing spondylitis concomitant with uveitis in a Han Chinese population," PLoS ONE, vol. 8, no. 6, Article ID e67505, 2013.

[11] J. Wagner, W. H. Sim, J. A. Ellis et al., "Interaction of crohn's disease susceptibility genes in an australian paediatric cohort," PLoS ONE, vol. 5, no. 11, Article ID e15376, 2010.

[12] E. Zintzaras and J. P. A. Ioannidis, "HEGESMA: genome search meta-analysis and heterogeneity testing," Bioinformatics, vol. 21, no. 18, pp. 3672-3673, 2005.

[13] E. Safrany, M. Szabo, M. Szell et al., "Difference of interleukin-23 receptor gene haplotype variants in ulcerative colitis compared to Crohn's disease and psoriasis," Inflammation Research, vol. 62, no. 2, pp. 195-200, 2013.

[14] M. Szabo, E. Safrany, B. Pazar et al., "Marked diversity of IL23R gene haplotype variants in rheumatoid arthritis comparing with Crohn's disease and ankylosing spondylitis," Molecular Biology Reports, vol. 40, no. 1, pp. 359-363, 2013.

[15] L. R. Ferguson, D. Y. Han, A. G. Fraser, C. Huebner, W. J. Lam, and A. R. Morgan, "IL23R and IL12B SNPs and haplotypes strongly associate with Crohn's disease risk in a New Zealand population," Gastroenterology Research and Practice, vol. 2010, Article ID 539461, 12 pages, 2010.
[16] M. Lauriola, G. Ugolini, S. Rivetti et al., "IL23R, NOD2/ CARD15, ATG16L1 and PHOX2B polymorphisms in a group of patients with Crohn's disease and correlation with subphenotypes," International Journal of Molecular Medicine, vol. 27, no. 3, pp. 469-477, 2011.

[17] M. Lacher, S. Schroepf, J. Helmbrecht et al., "Association of the interleukin-23 receptor gene variant rs11209026 with Crohn's disease in German children," Acta Paediatrica, vol. 99, no. 5, pp. 727-733, 2010.

[18] A. Latiano, O. Palmieri, M. R. Valvano et al., "Replication of interleukin 23 receptor and autophagy-related 16-like 1 association in adult-and pediatric-onset inflammatory bowel disease in Italy," World Journal of Gastroenterology, vol. 14, no. 29, pp. 4643-4651, 2008.

[19] A. Márquez, J. L. Mendoza, C. Taxonera et al., "IL23R and IL12B polymorphisms in Spanish IBD patients: no evidence of interaction," Inflammatory Bowel Diseases, vol. 14, no. 9, pp. 1192-1196, 2008.

[20] M. L. Baptista, H. Amarante, G. Picheth et al., "CARD15 and IL23R influences Crohn's disease susceptibility but not disease phenotype in a Brazilian population," Inflammatory Bowel Diseases, vol. 14, no. 5, pp. 674-679, 2008.

[21] W. G. Newman, Q. Zhang, X. Liu, C. I. Amos, and K. A. Siminovitch, "Genetic variants in IL-23R and ATG16L1 independently predispose to increased susceptibility to Crohn's disease in a Canadian population," Journal of Clinical Gastroenterology, vol. 43, no. 5, pp. 444-447, 2009.

[22] T. Okazaki, M. H. Wang, P. Rawsthorne et al., "Contributions of IBD5, IL23R, ATG16L1, and NOD2 to Crohn's disease risk in a population-based case-control study: evidence of gene-gene interactions," Inflammatory Bowel Diseases, vol. 14, no. 11, pp. 1528-1541, 2008.

[23] J. Oliver, B. Rueda, M. A. López-Nevot, M. Gómez-García, and J. Martín, "Replication of an association between IL23R gene polymorphism with inflammatory bowel disease," Clinical Gastroenterology and Hepatology, vol. 5, no. 8, pp. 977.e2-981.e2, 2007.

[24] C. A. Anderson, D. C. O. Massey, J. C. Barrett et al., "Investigation of Crohn's disease risk loci in ulcerative colitis further defines their molecular relationship," Gastroenterology, vol. 136, no. 2, pp. 523.e3-529.e3, 2009.

[25] A. Zwiers, L. Kraal, T. C. T. M. van de Pouw Kraan, T. Wurdinger, G. Bouma, and G. Kraal, "Cutting edge: a variant of the IL-23R gene associated with inflammatory bowel disease induces loss of microRNA regulation and enhanced protein production," Journal of Immunology, vol. 188, no. 4, pp. 15731577, 2012.

[26] N. Haroon, "Endoplasmic reticulum aminopeptidase 1 and interleukin-23 receptor in ankylosing spondylitis," Current Rheumatology Reports, vol. 14, no. 5, pp. 383-389, 2012.

[27] E. Sánchez, B. Rueda, J. L. Callejas et al., "Analysis of interleukin23 receptor (IL23R) gene polymorphisms in systemic lupus erythematosus," Tissue Antigens, vol. 70, no. 3, pp. 233-237, 2007.

[28] Y. H. Lee, Y. H. Rho, S. J. Choi et al., “The PTPN22 C1858T functional polymorphism and autoimmune diseases-a metaanalysis," Rheumatology, vol. 46, no. 1, pp. 49-56, 2007.

[29] N. J. Prescott, S. A. Fisher, C. Onnie et al., "A general autoimmunity gene (PTPN22) is not associated with inflammatory bowel disease in a British population," Tissue Antigens, vol. 66, no. 4, pp. 318-320, 2005. 


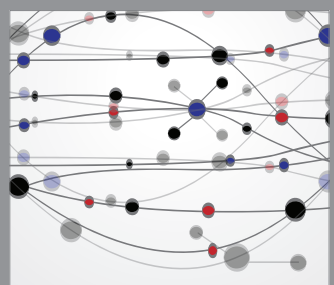

The Scientific World Journal
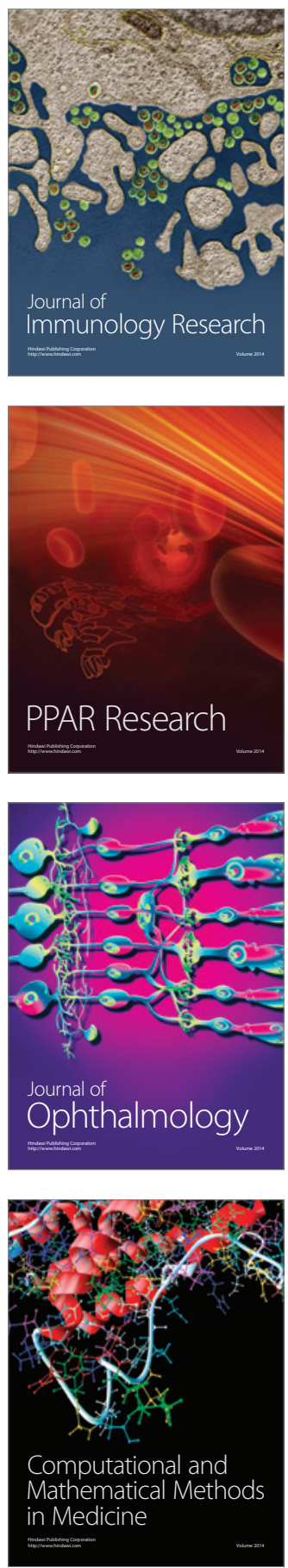

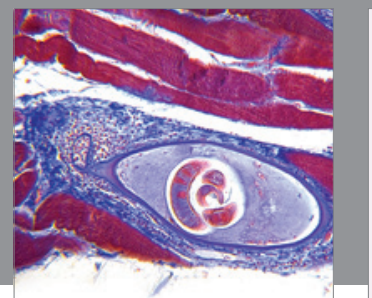

Gastroenterology

Research and Practice
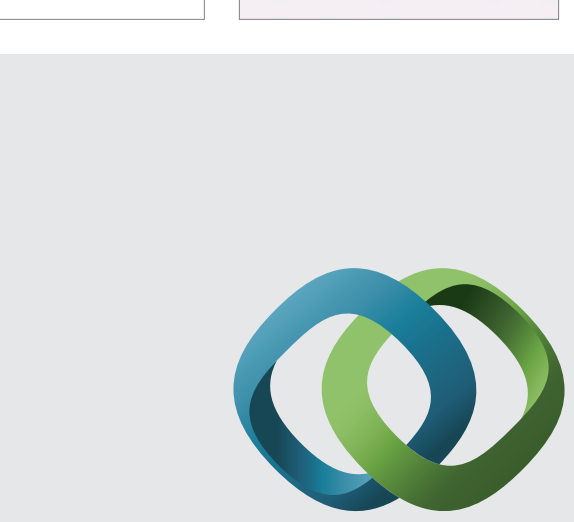

\section{Hindawi}

Submit your manuscripts at

http://www.hindawi.com
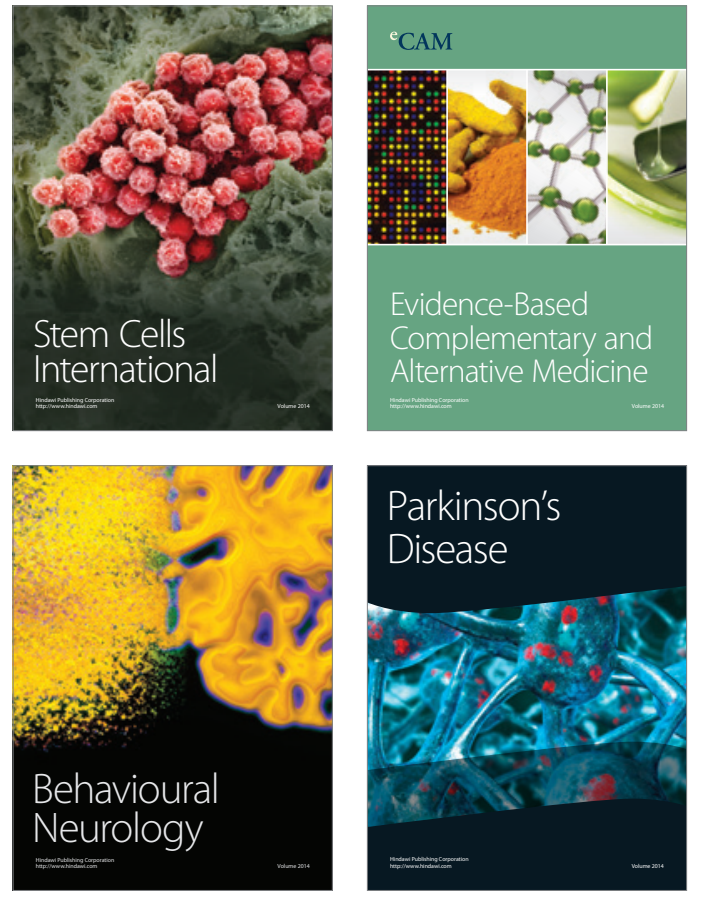
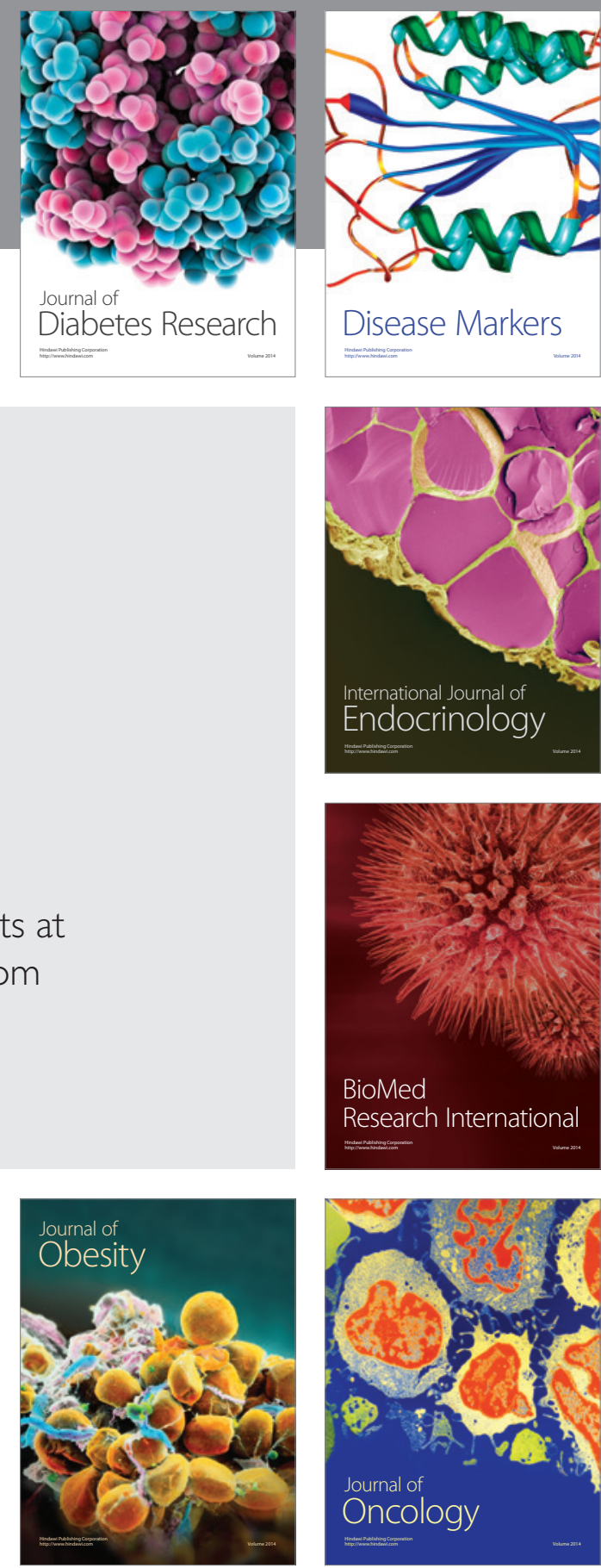

Disease Markers
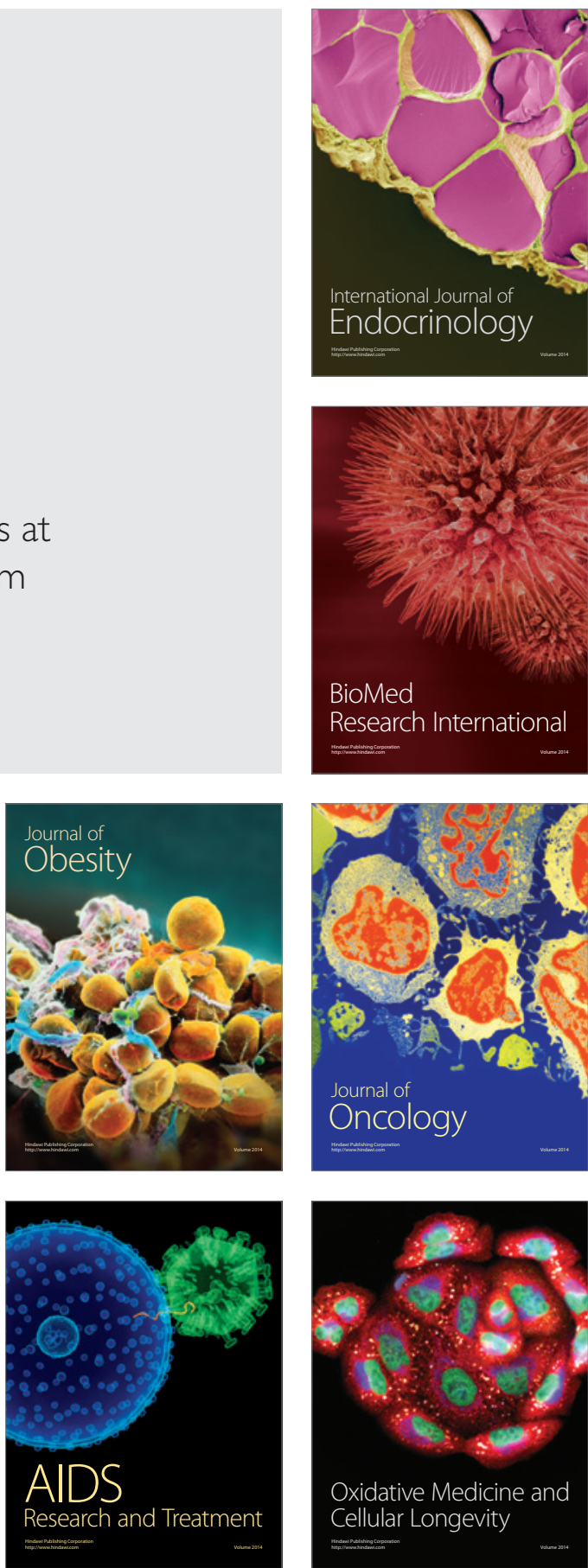